\title{
El marketing digital en la decisión de compra de los clientes de las empresas de electrodomésticos de la ciudad de Jaén
}

\author{
Mg. CPC. Víctor Hugo Puican Rodríguez \\ Victor-puican@hotmail.com \\ https://orcid.org/0000-0001-7402-9576 \\ Universidad Católica de Trujillo \\ Trujillo - Perú \\ Mg. Econ. Jorge Antonio Malca Florindes \\ mflorindesj@ucvvirtual.edu.pe \\ https://orcid.org/0000-0002-1090-6389
}

\section{RESUMEN}

Se utilizó como objetivo general: Determinar la relación entre el marketing digital y la decisión de compra de los clientes de las empresas de electrodomésticos de la ciudad de Jaén. Bajo un estudio con enfoque cuantitativo, aplicada, correlacional causal, no experimental de corte transversal, se utilizó el método estadístico descriptivo y el inferencial, encuestando a 380 clientes. Con respecto a los resultados el marketing digital alcanzó un 34\% en el nivel regular, asimismo, se evidenció que el marketing interno alcanzó un $67 \%$ en el nivel regular, en cambio, el marketing de contenidos obtuvo un $33 \%$ en el nivel deficiente. Asimismo, la decisión de compra alcanzó un 34\% en el nivel moderado, los factores sociales alcanzaron un $34 \%$ en el nivel moderado y los factores personales y Psicológicos alcanzaron un $65 \%$ en el nivel alto. Se alcanzó una significancia menor a 0.05 , demostrando que los resultados son estadísticamente muy significativos, del mismo modo se evidenció que el marketing digital mantiene una relación positiva moderada con la decisión de compra y con los factores sociales porque ambas alcanzaron un Rho de Spearman de 0.506 y 0.504 respectivamente, en cambio, los factores personales y Psicológicos mantienen una relación positiva alta porque alcanzaron un Rho de Spearman de 0.855 .

Palabras clave: marketing interno; marketing de contenidos; factores sociales; personales y psicológicos. 


\title{
Digital marketing in the purchase decision of customers of household appliances companies in the city of Jaén
}

\begin{abstract}
The general objective was: To determine the relationship between digital marketing and the purchase decision of customers of household appliances companies in the city of Jaén. Under a study with a quantitative approach, applied, causal correlational, nonexperimental cross-sectional, descriptive and inferential statistical method was used, surveying 380 customers. Regarding the results, digital marketing reached $34 \%$ in the regular level, likewise, it was evidenced that internal marketing reached $67 \%$ in the regular level, on the other hand, content marketing obtained $33 \%$ in the deficient level. Likewise, purchase decision reached $34 \%$ in the moderate level, social factors reached $34 \%$ in the moderate level and personal and psychological factors reached $65 \%$ in the high level. A significance of less than 0.05 was reached, demonstrating that the results are statistically very significant, in the same way it was evidenced that digital marketing maintains a moderate positive relationship with the purchase decision and with the social factors because both reached a Spearman's Rho of 0.506 and 0.504 respectively, on the other hand, the personal and Psychological factors maintain a high positive relationship because they reached a Spearman's Rho of 0.855 .
\end{abstract}

Keywords: internal marketing; content marketing; social; personal and psychological factors.

Artículo recibido: 05 octubre. 2021

Aceptado para publicación: 02 noviembre 2021 Correspondencia: Victor-puican@ hotmail.com

Conflictos de Interés: Ninguna que declarar 


\section{INTRODUCCIÓN}

Desde hace varias décadas venimos viviendo en un mundo fuertemente digitalizado y esto se ha fortalecido por el confinamiento social decretado por los gobiernos a nivel mundial con el propósito de parar el contagio del COVID-19, obligando a las empresas de Cuba a tener presencia online para que puedan seguir existiendo en el mercado. (Francisco, 2020) Estas situaciones ha ocasionado que el comportamiento en la decisión de compra de los clientes se modifique, debiendo las organizaciones utilizar el marketing digital para mantener un nivel de ventas estable que permita a la entidad mantener sus estados financieros en azul, que les permita afrontar sus pasivos corrientes en el plazo establecido y con ello obtener las utilidades que tanto anhelan (Duffus \& Briley, 2021). En cambio, en México las empresas no utilizan eficientemente el marketing digital y no se preocupan en promover la protección del medioambiente, generando disminución en sus ventas porque no se preocupan en conocer la decisión de compra de cada uno de sus clientes (Mehmood \& Tariq, 2021); Al transmitir mensajes de que las organizaciones se preocupan por proteger el medioambiente a través de la utilización del marketing digital se estará generando un efecto positivo en la actitud del cliente, llevándolo en lo posible tomar la decisión de adquirir nuestros productos o contratar nuestros servicios, a esto se le llama posicionar nuestra marca en la mente de cada cliente (Addo et al., 2021).

Asimismo, las empresas peruanas no utilizan herramientas y medios tecnológicos adecuados al momento de aplicar las estrategias de marketing generando un impacto negativo en la decisión de compra de los clientes, trayendo como consecuencia una pérdida de mercado considerable; siendo necesario que las empresas se interesen en conocer la importancia que posee el marketing digital en la decisión de compra de los clientes, porque, este acompaña al usuario en su búsqueda de los productos y servicios que necesita, permitiéndole interactuar y conocer las ofertas que la empresa ha planificado para cada uno de los clientes (Chávez et al., 2020).

Las empresas que se dedican a la comercialización de electrodomésticos en la ciudad de Jaén, han tenido que cerrar sus establecimientos por la reducción parcial o total de sus ventas. Un caso particular fue la empresa Elektra que tuvo que liquidar definitivamente sus actividades comerciales en esta provincia y a nivel nacional, siendo generado por la alta morosidad de sus clientes y por la disminución de sus ventas, demostrando que no han sabido evidenciar las enormes oportunidades que ha ocasionado el aislamiento social, 
porque con ello se han creado nuevas plataformas digitales que permiten interactuar en tiempo real con cada uno de sus clientes, como es el caso de muchas otras empresas en esta ciudad.

Con respecto a los trabajos previos se tomaron en cuenta los aportes de Pitre et al., (2020). Porque encontraron que las dificultades y barreras culturales que deben ser superadas por las pymes para su adecuado aprovechamiento son el uso inadecuado de las TIC. Asimismo, se consideró el estudio de Calle et al., (2020). Porque mencionan que la implementación de estrategias digitales integradas en un plan mejora el posicionamiento de marca, la imagen, el nivel de eficiencia y el posicionamiento de mercado en la empresa objeto de estudio. Del mismo modo, Verdín et al., (2020). Porque determinaron que los conocimientos que posee la empresa sobre marketing digital son insuficientes, siendo el principal motivo por el cual los potenciales clientes no toman la decisión de comprar sus productos.

Con respecto a las bases teóricas, se consideró para el marketing digital el enfoque organizacional, donde Chiavenato (2006). Menciona que son las relaciones que deben existir entre las funciones, niveles y actividades de los recursos materiales y humanos dentro de una organización, que le permitan alcanzar la eficiencia en todos los procesos de venta de sus productos o servicios a sus potenciales clientes, para ello es necesario que se combinen los conocimientos específicos, con las herramientas y tecnología aplicada en la ejecución de las estrategias que manejan en la entidad a través del marketing digital. Esta teoría hace referencia sobre alcanzar la eficiencia en todos los procesos que se ejecutan desde la adquisición o elaboración del producto o servicio, hasta su oferta y venta al cliente, es por ello que recomienda que, los empleados utilicen las tecnologías de información que les permita mantener una interacción activa con cada uno de sus compradores.

Con respecto a la conceptualización del marketing digital, se dice que es un grupo de estrategias destinadas hacia la promoción y difusión de una marca por internet. (Bricio, 2018) Asimismo, son las ideas creativas que elabora la organización para dar a conocer sus productos o servicios que ofrece, como también las ofertas y promociones, siendo comunicadas a través de todas las plataformas digitales que posee la institución. (Guamán et al., 2021) 
Las dimensiones consideradas en el marketing digital fueron, el marketing interno, donde se revela que para aumentar la productividad laboral dentro de la organización es esencial para poder cubrir las necesidades de la empresa con respecto al cambio del mercado. (Purwanti et al., 2021) Estas medidas permiten a la organización mostrar valor a sus objetivos, estrategias, estructuras, dirigentes y demás componentes a un mercado constituido por sus trabajadores, con el propósito de aumentar su productividad y lealtad por medio de un ambiente de trabajo adecuado que se interese por sus necesidades y deseos. (Ponzoa \& Erdmann, 2021)

Asimismo, debe considerar el desarrollo a las acciones relacionadas con la formación y desarrollo de habilidades, la orientación al cliente y la adquisición de nuevos conocimientos en cada uno de sus empleados; de la misma forma se debe realizar la contratación de empleados respetando los procesos de reclutamiento, selección y contratación debiendo ser dedicados y efectivos. (Dash \& Chakraborty, 2021)

Debe en lo posible realizar que sus empleados mantengan una adecuación en su trabajo debiendo ajustar las motivaciones y habilidades de cada integrante a las funciones y roles que se debe cumplir dentro de la organización, debiendo para ello, brindar empoderamiento dentro de su cargo y reconocer formalmente el alcance de sus objetivos. (Peter \& Dalla, 2020) Por último, se debe trabajar la comunicación interna, ya que, a través de esto se llega a socializar a los colaboradores con todos los niveles jerárquicos, informándoles constantemente sobre los objetivos y metas organizacionales que se deben cumplir para alcanzar los resultados, debiendo hacer entrega los valores institucionales, la cultura organizacional y los cambios que se produzcan a nivel interno y externo. (Anjala et al., 2021)

La segunda dimensión es el marketing de contenidos, el cual, se fundamenta sobre la idea de que al proporcionar contenido relevante y de valor añadido, nos posicionamos en la mente del consumidor como líderes de pensamiento y expertos en la industria donde operamos, provocando un acercamiento a la marca y acompañando al usuario en cualquiera de las fases del proceso que nos conduzca a nuestros objetivos. (Broekhuizen et al., 2021)

En primer lugar, invertirá gran parte de su tiempo entendiendo el mercado. (Faradillah et al., 2020) Es decir, comprendiendo las preferencias de los consumidores, sus hábitos de 
consumo en el ámbito de actuación y los contenidos aportados por otros actores de la industria. (Hamilton, 2020)

Una vez asumida esta base, procederá a la creación de contenido relevante y de calidad, con el objetivo de aportar el máximo valor posible a los consumidores. (Magano, 2020) Esto incluye mantener un equilibrio entre ofrecer cobertura a una amplia gama de temáticas, dentro de la industria en la que opere y la especialización en ciertas áreas, todo ello con la intención de atraer a un público más amplio. (Pandey et al., 2020)

El siguiente paso, como es obvio, será difundir el contenido por todo el ecosistema online y en paralelo promoverlo a través de las plataformas en las que no solo sea fácil encontrar el contenido, sino también compartirlo. (Alghizzawi, 2019)

Se debe ofrecer a los consumidores contenido de calidad, relevante y de valor en una sociedad digitalizada e interconectada como la actual, no solo significa aportar valor directamente a nuestro sitio web, sino transversalmente a una amplia gama de medios online diferentes (incluyendo: newsletters, libros blancos, webcasts/webinars, podcasts, vídeos, imágenes, foros, correo electrónico, blogs, redes sociales, redes de network), garantizando, eso sí, que ese contenido pueda ser encontrado fácilmente mediante la utilización de una sólida estrategia de Search Engine Optimization (SEO). (Bartosik, 2019)

Con respecto a la variable decisión de compra, se consideró el enfoque de las necesidades de Maslow, donde Lussier y Achua (2005), revelan que cada ser humano posee diferentes necesidades, dependiendo de su cultura, de sus costumbres y del estilo de vida que posea dentro de la sociedad, pero al final todos tenemos necesidades fisiológicas, como el hambre y la sed, también tenemos necesidades de seguridad y protección, es por ello que, cuando la empresa se gana la confianza de sus clientes, tendrán mayores compras en paralelo, porque, estos recomendarán los productos y servicios de la organización. De la misma forma todos los individuos poseen necesidades sociales, como sentimientos de pertenencia y amor, debiendo para ello, las organizaciones mantener un respeto y empatía con cada uno de sus visitantes compren o no sus productos, ya que, mediante ello podrán mantener una relación de cordialidad y confianza con cada uno de los visitantes. Asimismo, los individuos tenemos necesidades de estima, como de autoestima, estatus y reconocimiento, ya que, a través de ello, se sienten importantes en un grupo de amigos, familiar o de trabajo, debiendo para ello las organizaciones hacer público la premiación 
de los clientes ganadores, previa autorización del cliente. Por último, los individuos poseen necesidades de autorrealización, como la realización personal, en este punto la organización debe preocuparse en tener un plan de incentivos, de capacitación y de premios para todos sus trabajadores que obtengan la mejor productividad en el mes, con el fin de motivar a todos a realizar un mejor desempeño laboral.

Con respecto a la conceptualización de la decisión de compra, se revela que son los diferentes periodos previos por los que pasa un consumidor desde que nota la necesidad de comprar un determinado producto hasta que finalmente lo hace. (Al-Abdallah et al., 2021) Es la evolución por las distintas fases por las que pasa el consumidor y que van desde que reconoce que tiene una necesidad hasta que finalmente decide comprar. (Poushneh, 2021)

Como dimensiones de la decisión de compra se consideró a los factores sociales, donde se menciona que son los diversos hechos sociales determinar también el comportamiento del consumidor; la familia y grupos a los que pertenezca, así como el rol y estatus que tenga en esos grupos. (Gunawan et al., 2021) Se les llama grupos de referencia, a los grupos que el comprador actúa y que influyen sobre su comportamiento de compra, como son sus amigos, compañeros de trabajo, grupos religiosos o profesionales. (Rachmawati et al., 2020)

Tenemos al grupo familia, en este grupo, las necesidades y demandas de consumo se ven afectadas por los diferentes comportamientos de los diversos tipos de familia. (Woosik et al., 2020) Los roles de estos individuos dentro de un grupo influyen en su comportamiento de compra y en el de sus compañeros. (Mustafa \& Al-Abdallah, 2020) En cambio, en el estatus, se refiere al respeto o aprecio que se le tiene a aquella persona que goza de un prestigio entre los miembros del grupo, ya que, puede dar recomendaciones que los demás tendrán en cuenta. (Ali, 2019)

La segunda dimensión son los factores personales y psicológicos, en este punto se toma en cuenta la edad, fase de ciclo de vida de las personas, a lo largo de su vida de compras de diferentes bienes, porque sus gustos y hábitos cambian. (Pedreschi \& Nieto, 2021) De la misma forma nos enfocamos en su ocupación, sobre cuál es su tipo de trabajo del individuo, su estilo de vida, donde sus intereses, opiniones y actividades influyen al momento de comprar. (Cueva et al., 2021) Asimismo, se debe considerar las circunstancias económicas, porque, si hay crecimiento económico, las posibilidades de 
compra aumentan la idea es que satisfaga sus necesidades; de la misma manera se debe tomar en cuenta su personalidad, porque, cada individuo mantiene una personalidad diferente, algunos son autónomos, seguros y un alto nivel de sociabilidad, todas estas formas influyen en su comportamiento de compra. (Ramadan et al., 2019)

Se enmarca en la motivación, porque, las personas compran algún producto porque desean satisfacer algunas necesidades de distinta índole; de la misma manera se enfoca en el aprendizaje, el cual, mediante esto aumentan su experiencia y cambian su comportamiento de compra, seguidamente tenemos a la percepción, donde los individuos son diferentes, percibiendo la misma realidad pero de forma muy diferente, prestando atención a lo que le interesa, por último, tenemos a las convicciones y actitudes, esto hace mención que, con el aprendizaje los individuos adoptan nuevas creencias y comportamientos respecto al hecho de comprar. (Dobrucali, 2018)

\section{ESTRATEGIAS METODOLÓGICAS}

Se consideró utilizar una investigación aplicada, porque este tipo de trabajos permite la solución de los fenómenos prácticos encontrados mediante el uso de la teoría y de todos los conocimientos básicos de cada una de las variables de estudio. (CONCYTEC, 2018). Del mismo modo, se consideró utilizar un nivel de investigación correlacional causal, porque se decidió verificar el grado de relación que existe entre el marketing digital y la decisión de compra. (Vásquez, 2020).

El diseño utilizado fue el no experimental de corte transversal. Asimismo, se consideró determinar la relación entre el marketing digital y la decisión de compra de los clientes de las empresas de electrodomésticos de la ciudad de Jaén. Para ello, se consideró encuestar a 380 clientes de todas las empresas de electrodomésticos de la ciudad de Jaén. Para alcanzar los resultados se utilizaron los métodos estadísticos descriptivo e inferencial. Con respecto al método estadístico descriptivo se obtuvo una tabla con frecuencias y porcentajes por cada variable de estudio y de cada una de sus dimensiones, en cambio, en el método estadístico inferencial, se obtuvo la prueba de normalidad de datos, permitiendo contrastar las hipótesis y medir el grado de relación de las variables y de cada una de sus dimensiones. 
Para elaborar la encuesta se tomó en cuenta la siguiente información:

\begin{tabular}{|c|c|c|c|}
\hline 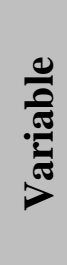 & 异 & Indicadores & Estrategia utilizada \\
\hline \multirow{11}{*}{ 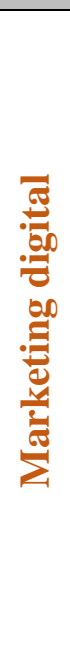 } & \multirow{7}{*}{ 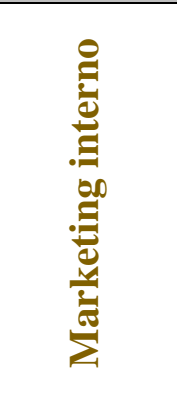 } & Productividad laboral & \multirow{24}{*}{$\begin{array}{l}\text { Para aplicar la encuesta, se } \\
\text { solicitó la lista de los clientes a } \\
05 \text { empresas que se dedican a la } \\
\text { venta de electrodomésticos en } \\
\text { la ciudad de Jaén, } \\
\text { posteriormente se contactó a } \\
\text { cada uno de ellos. Los que } \\
\text { aceptaron participar en la } \\
\text { encuesta fueron } 420 \text { clientes, se } \\
\text { les envío el link a su WhatsApp } \\
\text { de la encuesta virtual, a otros se } \\
\text { les envió por correo, donde solo } \\
\text { llegaron a contestar } 380 \\
\text { clientes, siendo esa cantidad } \\
\text { considerada como muestra de } \\
\text { estudio. }\end{array}$} \\
\hline & & Necesidad organizacional & \\
\hline & & Valor agregado & \\
\hline & & Ambiente de trabajo & \\
\hline & & Habilidades gerenciales & \\
\hline & & Orientación al cliente & \\
\hline & & Procesos de contratación & \\
\hline & & Contenido relevante & \\
\hline & 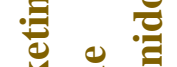 & Posicionamiento & \\
\hline & $\stackrel{y}{=} \approx$ & Marcas de productos & \\
\hline & & Fases del proceso & \\
\hline \multirow{13}{*}{ 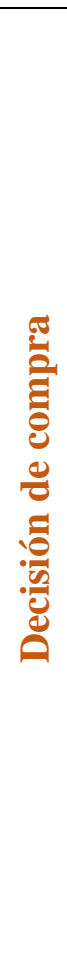 } & \multirow{6}{*}{ 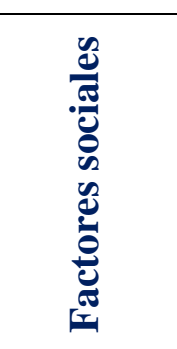 } & Hechos sociales & \\
\hline & & Comportamiento de compra & \\
\hline & & Grupos sociales & \\
\hline & & Demandas de consumo & \\
\hline & & Necesidades del cliente & \\
\hline & & Estatus del cliente & \\
\hline & \multirow{7}{*}{ 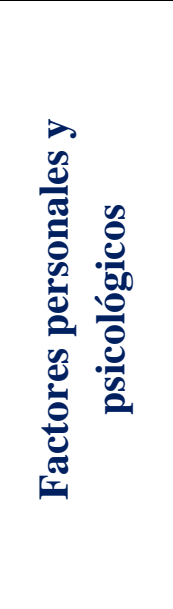 } & Datos históricos de compra & \\
\hline & & Intereses del cliente & \\
\hline & & Opiniones del cliente & \\
\hline & & $\begin{array}{l}\text { Actividades que realiza el } \\
\text { cliente }\end{array}$ & \\
\hline & & $\begin{array}{l}\text { Autonomía, seguridad } \quad \text { y } \\
\text { sociabilidad }\end{array}$ & \\
\hline & & $\begin{array}{l}\text { Experiencia } \quad \text { con } \quad \text { la } \\
\text { organización }\end{array}$ & \\
\hline & & $\begin{array}{lll}\text { Conocimiento } & \text { de } & \text { la } \\
\text { organización } & & \end{array}$ & \\
\hline
\end{tabular}




\section{RESULTADOS Y DISCUSIÓN}

\subsection{Resultados}

\subsubsection{Resultados descriptivos}

\section{Tabla 1}

Nivel de marketing digital de las empresas de electrodomésticos de la ciudad de Jaén.

\begin{tabular}{lcccccc}
\hline \multicolumn{1}{c}{\begin{tabular}{c}
\multicolumn{1}{c}{ Variable / } \\
Dimensiones
\end{tabular}} & \multicolumn{2}{c}{ Deficiente } & Regular & \multicolumn{3}{c}{ Eficiente } \\
\cline { 2 - 8 } Marketing digital & 126 & 33 & 130 & 34 & 124 & 33 \\
$\begin{array}{l}\text { Marketing interno } \\
\begin{array}{l}\text { Marketing de } \\
\text { contenidos }\end{array}\end{array}$ & 2 & 1 & 254 & 67 & 124 & 32 \\
\hline
\end{tabular}

Con respecto a los resultados del objetivo específico 1, se observa en la Tabla 1, que la variable marketing digital alcanzó un $34 \%$ en el nivel regular, pero en los niveles deficiente y eficiente alcanzó 33\% respectivamente, asimismo, se evidencia que la dimensión con peores resultados fue el marketing interno con un $67 \%$ en el nivel regular, en cambio, la dimensión con mejores resultados fue el marketing de contenidos con un $34 \%$ en el nivel eficiente, pero aún posee mayor porcentaje en los niveles deficiente y regular.

\section{Tabla 2}

Nivel de decisión de compra de los clientes de las empresas de electrodomésticos de la ciudad de Jaén

\begin{tabular}{lcccccc}
\hline \multirow{2}{*}{ Variable / Dimensiones } & \multicolumn{2}{c}{ Bajo } & \multicolumn{2}{c}{ Moderado } & \multicolumn{2}{c}{ Alto } \\
\cline { 2 - 7 } & f & \% & f & \% & f & \% \\
\hline Decisión de compra & 126 & 33 & 129 & 34 & 125 & 33 \\
Factores sociales & 127 & 33 & 129 & 34 & 124 & 33 \\
$\begin{array}{l}\text { Factores personales y } \\
\text { Psicológicos }\end{array}$ & 2 & 1 & 128 & 34 & 250 & 65 \\
\hline
\end{tabular}

Con respecto al objetivo específico 2, se evidencia en la Tabla 2, que la variable decisión de compra alcanzó un 34\% en el nivel moderado, asimismo, alcanzó un 33\% en los niveles bajo y alto, en cambio, en la dimensión que peores resultados obtuvo fue en los factores sociales porque alcanzó un 34\% en el nivel moderado y un 33\% en el nivel bajo, 
en cambio, la dimensión con mejores resultados fueron los factores personales y Psicológicos con un $65 \%$ en el nivel alto.

\subsubsection{Resultados inferenciales}

\section{Tabla 3}

Relación del marketing digital con la decisión de compra de los clientes de las empresas de electrodomésticos de la ciudad de Jaén

\begin{tabular}{|c|c|c|c|c|c|c|}
\hline \multicolumn{2}{|c|}{ MD } & DC & FS & FPS & MI & MC \\
\hline DC &, $506^{* *}$ & & & & & \\
\hline FS &, $504^{* * *}$ &, $996^{* *}$ & & & & \\
\hline FPS &, $855^{* *}$ &, $857^{* *}$ &, $860^{* *}$ & & & \\
\hline MI &, $862^{* *}$ &, $687^{* *}$ &, $860^{* *}$ &, $509^{* *}$ & & \\
\hline $\mathrm{MC}$ &, $498^{* *}$ &, $980^{* *}$ & $745^{* *}$ &, $833^{* *}$ &, $509^{* *}$ & 1,000 \\
\hline
\end{tabular}

Nota: MD: Marketing digital. DC: Decisión de compra. FS: Factores sociales. FPS: Factores personales y Psicológicos. MI: Marketing interno. MC: Marketing de contenidos.

De acuerdo a los datos alcanzados a través de la prueba de normalidad se alcanzó una significancia menor a 0.05 , demostrando que los resultados son estadísticamente muy significativos, asimismo, se utilizó el Rho de Spearman, donde reveló que la variable marketing digital mantiene una relación positiva moderada con la decisión de compra y con su dimensión factores sociales porque ambas alcanzaron un 0.506 y 0.504 respectivamente, en cambio, con la dimensión factores personales y Psicológicos mantiene una relación positiva alta porque alcanzó un Rho de Spearman de 0.855.

\subsection{DISCUSIÓN}

Los resultados alcanzados en el primer objetivo específico, se identificó que la variable marketing digital alcanzó un 34\% en el nivel regular, pero en los niveles deficiente y eficiente alcanzó $33 \%$ respectivamente, asimismo, se evidencia que la dimensión con peores resultados fue el marketing interno con un $67 \%$ en el nivel regular, en cambio, la dimensión con mejores resultados fue el marketing de contenidos con un 34\% en el nivel eficiente, pero aún posee mayor porcentaje en los niveles deficiente y regular.

Esto demuestra que la productividad laboral en estas organizaciones no es la adecuada, porque no llegan a cubrir las necesidades de la empresa con las ventas que se están obteniendo, asimismo, se observa que no se está dando el valor adecuado a los objetivos 
estratégicos que la empresa ha planteado en su POI, ocasionando que el ambiente de trabajo sea inadecuado, impactando negativamente cuando estos atienden a los clientes sea por teléfono, por las redes sociales o en las mismas agencias de ventas. Es por ello que es sumamente importante que se modifiquen las políticas de reclutamiento, selección y contratación de personal, donde no solo de debe evaluar el conocimiento, sino, también las habilidades de interactuar en un tiempo corto con personas desconocidas, porque esto permite observar si este empleado podrá desenvolverse de forma efectiva ante alguna situación adversa.

De la misma manera se evidencia que este tipo de organizaciones no cuentan con un plan de contingencia ante los hechos sociales que vienen ocurriendo, siendo uno de los factores que ha ocasionado que las ventas disminuyan, que la empresa no logre posicionarse en la mente del consumidor, todo esto ha afectado a las fases del proceso de compra que llevan a cabo los clientes, debiendo de forma inmediata actualizar las preferencias de cada uno de los clientes, en el caso que estas empresas aún deseen continuar de forma activa en el mercado.

Con respecto al segundo objetivo específico, se identificó que la variable decisión de compra alcanzó un 34\% en el nivel moderado, asimismo, alcanzó un 33\% en los niveles bajo y alto, en cambio, en la dimensión que peores resultados obtuvo fue en los factores sociales porque alcanzó un $34 \%$ en el nivel moderado y un $33 \%$ en el nivel bajo, en cambio, la dimensión con mejores resultados fueron los factores personales y Psicológicos con un $65 \%$ en el nivel alto.

Estos datos revelan que este tipo de empresas no manejan un sistema que les permita conocer cuál es el comportamiento de compra que poseen sus clientes, tampoco conocen a qué grupos religiosos, profesionales, familiares y sociales pertenecen, desconociendo de esta forma los intereses, opiniones y actividades que estas personas realizan. La falta de datos históricos de las compras que han realizado los clientes, ha ocasionado que no se identifiquen de forma clara las necesidades de los compradores, ocasionando malas experiencias con este tipo de organizaciones.

Con respecto al objetivo general, a través de la prueba de normalidad se alcanzó una significancia menor a 0.05 , demostrando que los resultados son estadísticamente muy significativos, asimismo, se utilizó el Rho de Spearman, donde reveló que la variable marketing digital mantiene una relación positiva moderada con la decisión de compra y 
con su dimensión factores sociales porque ambas alcanzaron un 0.506 y 0.504 respectivamente, en cambio, con la dimensión factores personales y Psicológicos mantiene una relación positiva alta porque alcanzó un Rho de Spearman de 0.855.

Estos datos demuestran que al utilizar el marketing digital de forma adecuada y de acuerdo a las exigencias del mercado y de las necesidades de sus clientes entonces se puede influenciar en la decisión de compra de cada uno de los compradores.

\section{CONCLUSIÓN O CONSIDERACIONES FINALES}

- Se concluye que la variable marketing digital alcanzó un 34\% en el nivel regular, pero en los niveles deficiente y eficiente alcanzó 33\% respectivamente, asimismo, se evidencia que la dimensión con peores resultados fue el marketing interno con un $67 \%$ en el nivel regular, en cambio, la dimensión con mejores resultados fue el marketing de contenidos con un $34 \%$ en el nivel eficiente, pero aún posee mayor porcentaje en los niveles deficiente y regular. El marketing digital se ha convertido en una herramienta indispensable para que las organizaciones puedan dar a conocer sus productos, servicios, ofertas y promociones, permitiendo a las entidades alcanzar sus objetivos institucionales.

- Se concluye que la variable decisión de compra alcanzó un 34\% en el nivel moderado, asimismo, alcanzó un 33\% en los niveles bajo y alto, en cambio, en la dimensión que peores resultados obtuvo fue en los factores sociales porque alcanzó un $34 \%$ en el nivel moderado y un $33 \%$ en el nivel bajo, en cambio, la dimensión con mejores resultados fueron los factores personales y Psicológicos con un $65 \%$ en el nivel alto. Para poder influenciar en la decisión de compra las organizaciones deben elaborar contenido de valor que permita trabajar las emociones de cada cliente, debiendo crear una relación de respeto y honestidad, debiendo siempre ser sincero al momento de transmitir información de los productos y servicios.

- Se concluye que se alcanzó una significancia menor a 0.05 , demostrando que los resultados son estadísticamente muy significativos, asimismo, se utilizó el Rho de Spearman, donde reveló que la variable marketing digital mantiene una relación positiva moderada con la decisión de compra y con su dimensión factores sociales porque ambas alcanzaron un 0.506 y 0.504 respectivamente, en cambio, con la dimensión factores personales y Psicológicos mantiene una relación positiva alta porque alcanzó un Rho de Spearman de 0.855. 


\section{LISTA DE REFERENCIAS}

Addo, P., Fang, J., Ohemeng, A., \& Kulbo, N. (2021). Customer engagement and purchase intention in live-streaming digital marketing platforms. The Service Industries $\quad$ Journal, $\quad$ 767-786. https://doi.org/10.1080/02642069.2021.1905798

Al-Abdallah, G., Khair, N., \& Elmarakby, R. (2021). The Impact of Social Networking Sites on Luxury Vehicles Purchase Decision Process in Gulf Cooperation Council Countries. Journal of International Consumer Marketing, 33(5), 559-577. https://doi.org/10.1080/08961530.2020.1867023

Alghizzawi, M. (2019). The role of digital marketing in consumer behavior:A survey. International Journal of Information Technology and Language Studies, 3(1), 2431.

https://www.researchgate.net/profile/Mahmoud-

Alghizzawi/publication/332593102_The_role_of_digital_marketing_in_consum er_behavior_A_survey/links/5cbf533e4585156cd7ad23c0/The-role-of-digitalmarketing-in-consumer-behavior-A-survey.pdf

Ali, H. (2019). Purchase Decision and Repurchase Models: Product Quality and Process Analysis (Case Study of House Ownership Credit Financing in Permata Sharia Bank Jakarta). Scholars Bulletln, 1(1), 526-535.

10.36348/sb.2019.v05i09.006

Anjala, S., Yogesh, K., Bindu, N., \& Satheesh, K. (2021). A broad overview of interactive digital marketing: A bibliometric network analysis. Journal of Business Research, 131(1), 183-195. https://doi.org/10.1016/j.jbusres.2021.03.061

Bartosik, M. (2019). Digital Marketing Communication from the Perspective of Individual Consumers: A Cross-Country Comparison. Entrepreneurial Business and Economics Review, 16(3), 205-220.

https://www.ceeol.com/search/article-detail?id=976259

Bricio, K. (2018). El marketing digital: Como herramienta en el desempeño laboral en el entorno ecuatoriano estudio de caso de los egresados de la Universidad de Guayaquil. /Universidad y Sociedad, 10(4), 103-109.

http://rus.ucf.edu.cu/index.php/rus 
Broekhuizen, T., Emrich, O., Gijsenberg, M., Broekhuis, M., Donkers, B., \& Sloot, L. (2021). Digital platform openness: Drivers, dimensions and outcomes. Journal of Business Research, 122(1),

902-914. https://doi.org/10.1016/j.jbusres.2019.07.001

Calle, K., Erazo, J., \& Narváez, C. (2020). Marketing digital y estrategias online en el sector de fabricación de muebles de madera. Revista Arbitrada Interdisciplinada Koinonía, 5(10), 26. .

https://doi.org/10.35381/r.k.v5i10.698

Chávez, E., Cruz, G., Zirena, P., \& De la Gala, R. (2020). Social media influencer: Influencia en la decisión de compra de consumidores millennial, Arequipa, Perú. Revista Venezolana de Gerencia, 25(3), 299-315. https://www.researchgate.net/profile/Patricia-Zirena-

Bejarano/publication/343999906_Social_media_influencer_Influencia_en_la_de cision_de_compra_de_consumidores_millennial_Arequipa_Peru/links/5f7bf5d 2 92851c14bcb1648d/Social-media-influencer-Influencia-en-1

Chiavenato, I. (2006). Introducción a la teoría general de la administración (7ma ed.). McGraw - Hill Interaméricana.

CONCYTEC. (2018). Investigación aplicada. https://portal.concytec.gob.pe/images/renacyt/reglamento_renacyt_version_final. pdf

Cueva, J., Sumba, N., \& Duarte, W. (2021). Marketing de contenidos y decisión de compra de los consumidores Generación Z en Ecuador. ECA SINERGIA, 12(2), 25-37.

https://doi.org/10.33936/eca_sinergia.v12i2.3459

Dash, G., \& Chakraborty, D. (2021). Digital Transformation of Marketing Strategies during a Pandemic: Evidence from an Emerging Economy during COVID-19. Digital Transformation of Marketing Strategies during a Pandemic: Evidence from an Emerging Economy during COVID-19, 13(12), 1-19. https://doi.org/10.3390/su13126735

Dobrucali, B. (2018). Country-of-origin effects on industrial purchase decision making: a systematic review of research. Journal of Business \& Industrial Marketing, 34(2), 401-411. 
https://doi.org/10.1108/JBIM-07-2017-0169

Duffus, D., \& Briley, D. (2021). Turista digital: variables que definen su comportamiento de compra. Revista Investigaciones Turísticas, 1(21), 1-21. . https://doi.org/10.14198/INTURI2021.21.1

Faradillah, I., Ummi, S., \& Nor, H. (2020). Digital Marketing: An Influence towards Business Performance among Entrepreneurs of Small and Medium Enterprises. International Journal of Academic Research in Business \& Social Sciences, 10(9), 126-141.

http://dx.doi.org/10.6007/IJARBSS/v10-i9/7709

Francisco, M. (2020). Marketing digital y el proceso de decisión de compra del consumidor habitual de vino. [Tesis de maestría, Instituto Superior de Contabilidades e Administracao]. https://recipp.ipp.pt/bitstream/10400.22/17765/1/Marina_Veintimilla_MMKD_2 020.pdf

Guamán, M., Paredes, J., \& Llivisaca, M. (2021). Marketing digital durante tiempos de COVID-19 en el sector comercial: caso Pichincha - Ecuador. Polo del conocimiento, 6(3), 497-519.

$10.23857 /$ pc.v6i3.2383

Gunawan, L., Haryono, S., \& Andreani, F. (2021). Social media influencer, brand awareness, and purchase decision among generation $\mathrm{z}$ in surabaya. Journal Manajemen dan Kewirausahaan, 23(1), 18-26. https://doi.org/10.9744/jmk.23.1.18-26

Hamilton, N. (2020). Evolving and enhanced dimensions of digital marketing strategies during contemporary scenario of COVID -19. International Journal of Multidisciplinary educational research, 9(8), 56-61. http://ijmer.s3.amazonaws.com/pdf/volume9/volume9-issue5(8)2020.pdf\#page $=65$

Hernández, H., Pitre, R., \& Builes, S. (2020). Impacto del marketing digital a las empresas colombianas emergentes. Universidad y Empresa, 23(40), 1-20. https://doi.org/10.12804/revistas.urosario.edu.co/empresa/a.9114

Lussier, R., \& Achua, C. (2005). Liderazgo: Teoría, aplicación y desarrollo de habilidades (2da. ed.). International Thomson Editores. 
Magano, J. (2020). Digital marketing impact on tourism in Portugal: a quantitative study. African Journal of Hospitality, Tourism and Leisure, 9(1), 1-19. https://www.researchgate.net/profile/Jose-

Magano/publication/338655966_Digital_marketing_impact_on_tourism_in_Port ugal_a_quantitative_study/links/5e21c41b92851cafc38c5f2b/Digital-marketingimpact-on-tourism-in-Portugal-a-quantitative-study.pdf

Mehmood, T., \& Tariq, N. (2021). Footprints of Digital Marketing on Customers' Purchase Decision. lectronic Research Journal of Social Sciences and Humanities , 3(1), 20-30.

https://papers.ssrn.com/sol3/papers.cfm?abstract_id=3775686

Mustafa, S., \& Al-Abdallah, G. (2020). The evaluation of traditional communication channels and its impact on purchase decision. Management Science Letters, 10(7), 1521-1532.

10.5267/j.msl.2019.12.014

Pandey, N., Nayal, P., \& Singh, A. (2020). Digital marketing for B2B organizations: structured literature review and future research directions. Journal of Business \& Industrial Marketing, 35(7), 1191-1204. https://doi.org/10.1108/JBIM-06-2019-0283

Pedreschi, R., \& Nieto, O. (2021). Efecto e impacto del neuromarketing en la decisión de compra de los estudiantes de la Facultad de Administración de Empresas y Contabilidad de la Extensión Universitaria de Aguadulce. Orbis Cógnita, 5(2), 111-128.

https://www.revistas.up.ac.pa/index.php/orbis_cognita/article/view/2324

Peter, M., \& Dalla, M. (2020). The Digital Marketing Toolkit: A Literature Review for the Identification of Digital Marketing Channels and Platforms. New Trends in Business Information Systems and Technology , 294(1), 251-265. https://link.springer.com/chapter/10.1007/978-3-030-48332-6_17

Ponzoa, J., \& Erdmann, A. (2021). E-Commerce Customer Attraction: Digital Marketing Techniques, Evolution and Dynamics across Firms. Journal of Promotion Management, 27(5), 697-715.

https://doi.org/10.1080/10496491.2021.1880521 
Poushneh, A. (2021). How close do we feel to virtual product to make a purchase decision? Impact of perceived proximity to virtual product and temporal purchase intention. Journal of Retailing and Consumer Services, 63(1), 1-11. https://doi.org/10.1016/j.jretconser.2021.102717

Purwanti, Y., Erlangga, H., KurniasIh, D., Pratama, A., Sunarsi, D., Nurjaya, . . . Purwanto, A. (2021). The Influence Of Digital Marketing \& Innovasion On The School Performance. Turkish Journal of Computer and Mathematics Education, 12(7),

118-127.

https://turcomat.org/index.php/turkbilmat/article/view/2551/2188

Rachmawati, E., Suliyanto, \& Suroro, A. (2020). A moderating role of halal brand awareness to purchase decision making. Journal of Islamic Marketing, 1(1), 1-9. https://doi.org/10.1108/JIMA-05-2020-0145

Ramadan, Z., Farah, M., \& Kassab, D. (2019). Amazon's approach to consumers' usage of the Dash button and its effect on purchase decision involvement in the U.S. market. Journal of Retailing and Consumer Services, 47(1), 133-139. https://doi.org/10.1016/j.jretconser.2018.11.018

Vásquez, W. A. (2020). Metodología de la investigación. https://www.usmp.edu.pe/estudiosgenerales/pdf/2019-

II/MANUALES/II\%20CICLO/METODOLOGIA\%20DE\%20LA\%20INVESTI GACION.pdf

Verdín, J., González, M., \& Casas, M. (2020). Influencia de la mercadotecnia digital en la decisión de compra del consumidor. Estudio de caso: Xicali-Cocina de humo. Vincula Tégica EFAN, $\quad$ 1(1), 298-304. http://www.web.facpya.uanl.mx/Vinculategica/Vinculategica6_1/22\%20VERDI N_GONZALEZ_CASAS.pdf

Woosik, D., Lee, S., \& Alcorn, M. (2020). Influence of culture on purchase decision: Integrative models development of amusement park customers. International Journal of Hospitality Management, $87(1), \quad$ 1-16. https://doi.org/10.1016/j.ijhm.2020.102502 